\title{
Crystal structure of $(E)-3^{\prime}, 6^{\prime}$-bis(ethylamino)-2', $7^{\prime}-$ dimethyl-2-((pyridin-2-ylmethylene)amino)spiro [isoindoline-1,9'-xanthen]-3-one, $\mathrm{C}_{32} \mathrm{H}_{31} \mathrm{~N}_{5} \mathrm{O}_{2}$
}

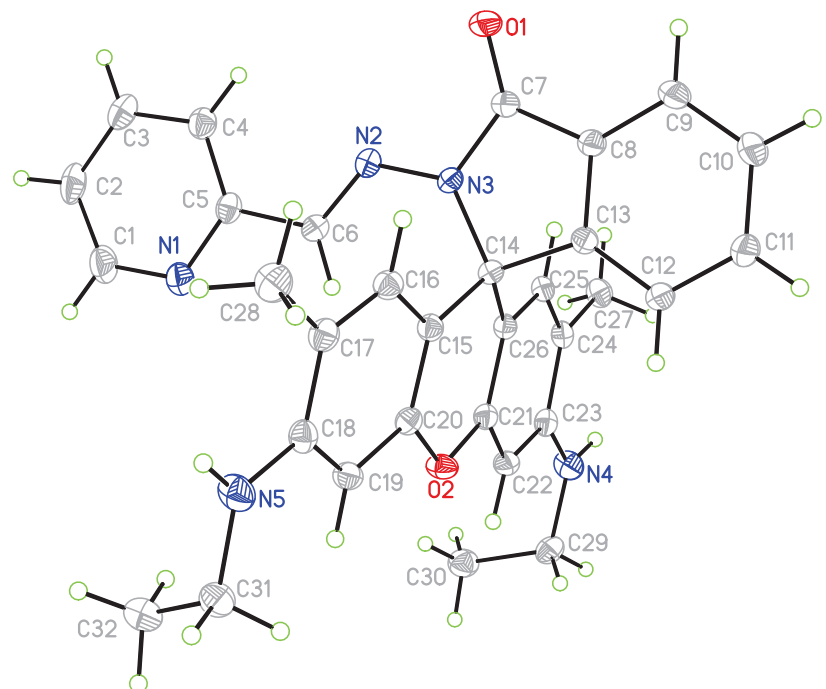

https://doi.org/10.1515/ncrs-2018-0239

Received July 13, 2018; accepted September 5, 2018; available online September 27, 2018

\section{Abstract}

$\mathrm{C}_{32} \mathrm{H}_{31} \mathrm{~N}_{5} \mathrm{O}_{2}$, triclinic, $P \overline{1} \quad$ (no. 2), $a=9.9226(4) \AA$, $b=11.1446(7) \AA, \quad c=12.6719(6) \AA, \quad \alpha=90.693(4)^{\circ}$, $\beta=109.858(4)^{\circ}, \quad \gamma=95.382(4)^{\circ}, \quad Z=2, \quad V=1310.72(12) \AA^{3}$, $R_{\mathrm{gt}}(F)=0.0497, w R_{\mathrm{ref}}\left(F^{2}\right)=0.1419, T=173(10) \mathrm{K}$.

CCDC no.: 1865970

The crystal structure is shown in the figure. Tables 1 and 2 contain details on crystal structure and measurement conditions and a list of the atoms including atomic coordinates and displacement parameters.

\section{Source of material}

All of the starting materials and solvents were reagent grade and were used without purification. The title compound was obtained from Rhodamine $6 \mathrm{G}$ by a two-step reaction.

\footnotetext{
*Corresponding author: Juan Yuan, Henan University of Chinese Medicine, Zhengzhou 450046, P.R. China, e-mail: hnzz_yuan@163.com

Di Cheng: Henan University of Chinese Medicine, Zhengzhou 450046, P.R. China
}

Table 1: Data collection and handling.

\begin{tabular}{ll}
\hline Crystal: & Colorless rod-like \\
Size: & $0.40 \times 0.30 \times 0.10 \mathrm{~mm}$ \\
Wavelength: & Cu $K \alpha$ radiation $(1.54184 \AA$ A $)$ \\
$\mu:$ & $0.67 \mathrm{~mm}^{-1}$ \\
Diffractometer, scan mode: & SuperNova, $\omega$ \\
$\theta_{\text {max }}$, completeness: & $76.8^{\circ},>99 \%$ \\
$N(h k l)_{\text {measured }}, N(h k l)_{\text {unique }}, R_{\text {int }}:$ & $8793,5295,0.024$ \\
Criterion for $I_{\text {obs }}, N(h k l)_{\text {gt }}:$ & $I_{\text {obs }}>2 \sigma\left(I_{\text {obs }}\right), 4778$ \\
$N(\text { param })_{\text {refined }}:$ & 356 \\
Programs: & OLEX2 [1], SHELX $[2,3]$, \\
& CrysAlis ${ }^{\text {PRO }[4]}$ \\
\hline
\end{tabular}

Table 2: Fractional atomic coordinates and isotropic or equivalent isotropic displacement parameters $\left(\AA^{2}\right)$.

\begin{tabular}{lrrrr}
\hline Atom & $\boldsymbol{x}$ & $\boldsymbol{y}$ & $\boldsymbol{z}$ & $\boldsymbol{U}_{\text {iso }}{ }^{*} \boldsymbol{U}_{\text {eq }}$ \\
\hline O2 & $0.62952(11)$ & $0.42340(9)$ & $0.52425(8)$ & $0.0301(2)$ \\
O1 & $0.64105(13)$ & $0.19506(11)$ & $0.99411(9)$ & $0.0408(3)$ \\
N3 & $0.66350(12)$ & $0.26114(10)$ & $0.82731(9)$ & $0.0257(2)$ \\
N2 & $0.78010(12)$ & $0.19989(11)$ & $0.84103(10)$ & $0.0280(3)$ \\
N4 & $0.30616(14)$ & $0.09943(13)$ & $0.30566(11)$ & $0.0367(3)$ \\
H4 & 0.2468 & 0.0372 & 0.3031 & $0.044^{*}$ \\
N5 & $1.00517(15)$ & $0.72836(13)$ & $0.71075(12)$ & $0.0399(3)$ \\
H5 & 1.0648 & 0.7613 & 0.7728 & $0.048^{*}$ \\
N1 & $1.03909(15)$ & $0.16870(14)$ & $0.71182(12)$ & $0.0423(3)$ \\
C26 & $0.53032(13)$ & $0.27276(12)$ & $0.62230(11)$ & $0.0241(3)$ \\
C20 & $0.71318(14)$ & $0.47944(12)$ & $0.62597(11)$ & $0.0257(3)$ \\
C15 & $0.70087(14)$ & $0.44633(12)$ & $0.72746(11)$ & $0.0251(3)$ \\
C13 & $0.47804(14)$ & $0.38260(12)$ & $0.77614(11)$ & $0.0255(3)$ \\
C25 & $0.44520(14)$ & $0.16212(12)$ & $0.60991(11)$ & $0.0264(3)$ \\
H25 & 0.4372 & 0.1275 & 0.6742 & $0.032^{*}$ \\
C21 & $0.54415(14)$ & $0.31894(12)$ & $0.52489(11)$ & $0.0248(3)$ \\
C14 & $0.59497(14)$ & $0.34197(12)$ & $0.73478(11)$ & $0.0241(3)$ \\
C22 & $0.47296(15)$ & $0.26201(13)$ & $0.41981(11)$ & $0.0278(3)$ \\
H22 & 0.4846 & 0.2958 & 0.3563 & $0.033^{*}$ \\
C23 & $0.38425(14)$ & $0.15456(13)$ & $0.40892(12)$ & $0.0279(3)$ \\
C19 & $0.81302(15)$ & $0.57225(13)$ & $0.61875(12)$ & $0.0290(3)$ \\
H19 & 0.8182 & 0.5919 & 0.5490 & $0.035^{*}$ \\
C24 & $0.37278(14)$ & $0.10188(13)$ & $0.50770(12)$ & $0.0277(3)$ \\
C16 & $0.79453(15)$ & $0.51148(13)$ & $0.82389(11)$ & $0.0286(3)$ \\
H16 & 0.7879 & 0.4916 & 0.8932 & $0.034^{*}$ \\
C7 & $0.60180(15)$ & $0.25416(13)$ & $0.91108(11)$ & $0.0289(3)$ \\
C17 & $0.89611(15)$ & $0.60368(13)$ & $0.82101(12)$ & $0.0307(3)$ \\
C18 & $0.90547(15)$ & $0.63610(13)$ & $0.71564(12)$ & $0.0299(3)$
\end{tabular}


Table 2 (continued)

\begin{tabular}{|c|c|c|c|c|}
\hline Atom & $x$ & $y$ & $z$ & $U_{\text {iso }} * / U_{\text {eq }}$ \\
\hline C5 & $0.97640(15)$ & $0.14832(13)$ & $0.78891(12)$ & $0.0307(3)$ \\
\hline $\mathrm{C} 8$ & $0.48382(15)$ & $0.33203(13)$ & $0.87663(11)$ & $0.0285(3)$ \\
\hline C6 & $0.84644(15)$ & $0.21117(13)$ & $0.77075(11)$ & $0.0286(3)$ \\
\hline H6 & 0.8134 & 0.2583 & 0.7089 & $0.034^{*}$ \\
\hline C12 & $0.37338(16)$ & $0.45832(14)$ & $0.72485(12)$ & $0.0312(3)$ \\
\hline H12 & 0.3688 & 0.4927 & 0.6574 & $0.037^{\star}$ \\
\hline $\mathrm{C} 4$ & $1.02854(17)$ & $0.07489(15)$ & $0.87850(13)$ & $0.0365(3)$ \\
\hline $\mathrm{H} 4 \mathrm{~A}$ & 0.9813 & 0.0628 & 0.9301 & $0.044^{*}$ \\
\hline $\mathrm{C} 27$ & $0.28226(16)$ & $-0.01633(14)$ & $0.49949(13)$ & $0.0336(3)$ \\
\hline $\mathrm{H} 27 \mathrm{~A}$ & 0.2895 & -0.0405 & 0.5735 & 0.050 * \\
\hline H27B & 0.3159 & -0.0767 & 0.4628 & $0.050^{*}$ \\
\hline $\mathrm{H} 27 \mathrm{C}$ & 0.1836 & -0.0072 & 0.4569 & $0.050^{*}$ \\
\hline C29 & $0.31836(17)$ & $0.14032(14)$ & $0.20117(12)$ & $0.0350(3)$ \\
\hline $\mathrm{H} 29 \mathrm{~A}$ & 0.3209 & 0.2275 & 0.2017 & $0.042^{\star}$ \\
\hline H29B & 0.2332 & 0.1076 & 0.1398 & $0.042^{*}$ \\
\hline C11 & $0.27577(18)$ & $0.48093(16)$ & $0.77781(14)$ & $0.0399(4)$ \\
\hline H11 & 0.2046 & 0.5311 & 0.7449 & $0.048^{*}$ \\
\hline C3 & $1.15248(18)$ & $0.01975(16)$ & $0.88987(15)$ & $0.0425(4)$ \\
\hline H3 & 1.1895 & -0.0304 & 0.9487 & $0.051^{\star}$ \\
\hline C9 & $0.38677(18)$ & $0.35506(16)$ & $0.93018(13)$ & $0.0388(4)$ \\
\hline H9 & 0.3921 & 0.3212 & 0.9980 & $0.047^{\star}$ \\
\hline $\mathrm{C} 28$ & $0.99480(18)$ & $0.67037(16)$ & $0.92720(14)$ & $0.0409(4)$ \\
\hline $\mathrm{H} 28 \mathrm{~A}$ & 0.9757 & 0.7533 & 0.9257 & $0.061^{*}$ \\
\hline $\mathrm{H} 28 \mathrm{~B}$ & 1.0931 & 0.6659 & 0.9328 & $0.061^{*}$ \\
\hline $\mathrm{H} 28 \mathrm{C}$ & 0.9785 & 0.6344 & 0.9908 & $0.061^{*}$ \\
\hline C10 & $0.28229(19)$ & $0.42992(17)$ & $0.87916(15)$ & $0.0431(4)$ \\
\hline H10 & 0.2156 & 0.4465 & 0.9127 & $0.052^{\star}$ \\
\hline C31 & $1.01471(18)$ & $0.77284(14)$ & $0.60603(15)$ & $0.0406(4)$ \\
\hline H31A & 1.0584 & 0.8557 & 0.6191 & $0.049^{\star}$ \\
\hline H31B & 0.9181 & 0.7726 & 0.5520 & $0.049^{\star}$ \\
\hline C30 & $0.4508(2)$ & $0.10433(16)$ & $0.17964(14)$ & $0.0425(4)$ \\
\hline $\mathrm{H} 30 \mathrm{~A}$ & 0.4540 & 0.1365 & 0.1105 & $0.064^{\star}$ \\
\hline H30B & 0.4465 & 0.0180 & 0.1746 & $0.064^{*}$ \\
\hline $\mathrm{H} 30 \mathrm{C}$ & 0.5358 & 0.1358 & 0.2403 & $0.064^{*}$ \\
\hline C2 & $1.21884(18)$ & $0.04142(19)$ & $0.81171(17)$ & $0.0498(4)$ \\
\hline $\mathrm{H} 2$ & 1.3024 & 0.0068 & 0.8169 & $0.060^{\star}$ \\
\hline C32 & $1.1013(2)$ & $0.69960(18)$ & $0.55593(16)$ & $0.0485(4)$ \\
\hline $\mathrm{H} 32 \mathrm{~A}$ & 1.0965 & 0.7294 & 0.4841 & $0.073^{*}$ \\
\hline H32B & 1.0623 & 0.6164 & 0.5468 & $0.073^{*}$ \\
\hline $\mathrm{H} 32 \mathrm{C}$ & 1.1998 & 0.7066 & 0.6052 & $0.073^{*}$ \\
\hline C1 & $1.1587(2)$ & $0.1158(2)$ & $0.72517(17)$ & $0.0531(5)$ \\
\hline 1 & 1.2046 & 0.1299 & 0.6729 & $0.064^{*}$ \\
\hline
\end{tabular}

Rhodamine $6 \mathrm{G}$ hydrozone is prepared according to the literature method [5]. To a $50 \mathrm{~mL}$ flask, Rhodamine $6 \mathrm{G}$ $(1 \mathrm{mmol}, 0.479 \mathrm{~g})$ was dissolved in $20 \mathrm{~mL}$ ethanol. $2.0 \mathrm{~mL}$ (excess) hydrazine monohydrate (80\%) was then added dropwise under stirring at room temperature. After the addition, the stirred mixture was refluxed for $3 \mathrm{~h}$, during which the pink precipitate appeared. The precipitate was filtered, washed 3 times with methanol/water (1:1). Yield: $c a 80 \%$.

The synthesis of the title compound. Rhodamine $6 \mathrm{G}$ hydrozone $(0.5 \mathrm{mmol}, 0.214 \mathrm{~g})$ was dissolved in boiling methanol, and 2-pyridinecarbaldehyde ( $0.5 \mathrm{mmol}, 0.5 \mathrm{~mL})$ was dropped to the above solution under stirring. Then, the mixture was refluxed for $5 \mathrm{~h}$ with vigorous stirring, and white precipitates appeared. The precipitate was filtered, washed 3 times with methanol/water (1:1) and dried over $\mathrm{P}_{2} \mathrm{O}_{5}$ under vacuum to obtain white powder. Yield: $c a$ $85 \%$. The white powder $(0.05 \mathrm{mmol}, 25.9 \mathrm{mg})$ was dissolved in methanol/dichloromethane (1:1) $(10 \mathrm{~mL})$ under stirring to give a colorless solution. Afterwards, ether $(20 \mathrm{~mL})$ was slowly diffused to the above solution in a sealed monotube. After 3 days, colourless rod-like crystals were obtained.

\section{Experimental details}

The $\mathrm{H}$ atoms were added using riding models. Their $U_{\text {iso }}$ values were set to $1.2 U_{\text {eq }}$ of the parent atoms.

\section{Comment}

Rhodamine derivatives have attracted great attention in the field of material and analytical chemistry [6-8], because of the excellent spectroscopic properties such as high fluorescence quantum yield and long wavelength emission. Rhodamine derivatives have been widely investigated as fluorescent probe and fluorescence chemosensors [9-11].

In this paper, we report a rhodamine $6 \mathrm{G}$ pyridyl aroylhydrozone compound. The asymmetric unit contains a neutral molecule in a ring-closed form. The amide $\mathrm{C}=\mathrm{O}$ bond distance is $1.2162(18) \AA$, indicative of the keto form. The aroylhydrozone plane and the pyridyl plane are nearly coplanar with the dihedral angle of $4.8^{\circ}$. The dihedral angle between the xanthene plane and pyridyl aroylhydrozone group is $84.8^{\circ}$.

Acknowledgements: This work was supported by the National Natural Science Foundation of China (Project No. 21571113) and the project of scientific and technological in Henan Province (Project No. 172102310433).

\section{References}

1. Dolomanov, O. V.; Bourhis, L. J.; Gildea, R. J.; Howard, J. A. K.; Puschmann, H.: OLEX2: a complete structure solution, refinement and analysis program. J. Appl. Crystallogr. 42 (2009) 339-341.

2. Sheldrick, G. M.: A short history of SHELX. Acta Crystallogr. A64 (2008) 112-122.

3. Sheldrick, G. M.: Crystal structure refinement with SHELXL. Acta Crystallogr. C71 (2015) 3-8.

4. Rigaku Oxford Diffraction Ltd., CrysAlis ${ }^{\mathrm{PRO}}$, Yarnton, Oxfordshire, UK (2015).

5. Yang, Y.-K.; Yook, K.-J.; Tae, J.: A rhodamine-based fluorescent and colorimetric chemodosimeter for the rapid detection of $\mathrm{Hg}^{2+}$ ions in aqueous media. J. Am. Chem. Soc. 127 (2005) 16760-16761. 
6. Yang, Y.; Zhao, Q.; Feng, W.; Li, F.: Luminescent chemodosimeters for bioimaging. Chem. Rev. 113 (2013) 192-270.

7. Chen, X. Q.; Pradhan, T.; Wang, F.; Kim, J. S.; Yoon, J.: Fluorescent chemosensors based on spiroring-opening of xanthenes and related derivatives. Chem. Rev. 112 (2012) 1910-1956.

8. Cheng, D.; Pan, Y.; Wang, L.; Zeng, Z. B.; Yuan, L.; Zhang, X. B.; Chang, Y.-T.: Selective visualization of the endogenous peroxynitrite in an inflamed mouse model by a mitochondriatargetable two-photon ratiometric fluorescent probe. J. Am. Chem. Soc. 139 (2017) 285-292.
9. Wu, D.; Huang, W.; Duan, C.; Lin, Z.; Meng, Q.: Probe for selective detection of $\mathrm{Hg}^{2+}$ in DMF aqueous media. Inorg. Chem. 46 (2007) 1538-1540.

10. Zhao, Y.; Zhang, X.-B.; Han, Z.-X.; Li, Q.; Li, C.-Y.; Jian, L.-X.; Shen, G.-L.; Yu, R.-Q.: Highly sensitive and selective colormetric and off-on fluorescent chemosensor for $\mathrm{Cu}^{2+}$ in aqueous solution and living cells. Anal. Chem. 81 (2009) 7022-7030.

11. Xiang, Y.; Tong, A.; Jin, P.; Ju, Y.: New fluorescent rhodamine hydrazine chemosensor for $\mathrm{Cu}(\mathrm{III})$ with high selectivity and sensitivity. Org. Lett. 8 (2006) 2863-2866. 